\title{
DIAGNÓSTICO SITUACIONAL DE LA PROBLEMÁTICA SANITARIA Y REPRODUCTIVA DE LA PRODUCCIÓN PECUARIA EN LA SIERRA DE LA PROVINCIA DE HUARAL
}

\author{
César Alzamora P. ${ }^{1}$, Juan Espinoza B. ${ }^{2}$, Felipe San Martín H. ${ }^{3}$ y \\ Luis Coronado S. ${ }^{1}$
}

\section{Evaluation of livestock health and reproduction in the highlands of Huaral province, Peru}

This paper describes livestock production systems in the highlands of Huaral Province, Peru, an area and topic about which little is known. Land ownership is distributed in smallholdings used primarily for agricultural production. Herding is the responsibility of men, most older than 50 years. Each small holder keeps from 1 to 20 cattle and 1 to 20 sheep, as well as pigs, poultry and guinea pigs. No annual preventative treatment schedule is followed and only a few producers have access to some level of technical advice. In most holdings, the animals are dosed annually for parasites, especially liver fluke. Reproduction is not controlled and animals are naturally mated. Placenta retention is the most important problem with cattle.

Key words: Animal health, reproduction, liver fluke, parasites, carbunco.

Se conoce muy poco sobre los sistemas de producción pecuarios de la sierra de la Provincia de Huaral de allí que el objetivo del presente estudio fue describir y diagnosticar el manejo sanitario y reproductivo de las especies pecuarias de la zona. Se prefiere el cultivo de alimentos al de forraje. Las tierras se distribuyen en pequeñas parcelas. La producción pecuaria es dirigida mayormente por los varones y más de la mitad de ellos tienen edades que superan los 50 años. La crianza de bovinos y ovinos varía de 1 a 20 animales por cada unidad agropecuaria para las dos especies. La crianza de otras especies son para auto abastecimiento (cerdos, aves de corral y cuyes). La sanidad no es preventiva sino curativa. El calendario de actividades sanitarias no se lleva a cabo debido a la ausencia de técnicos de campo. Un bajo porcentaje de productores cuenta con asistencia técnica la cual es brindada por un técnico agropecuario. Los productores llaman técnico a la persona que consultan sin saber de su real capacidad académica. La párticipación del técnico es calificada como regular. En la mayor parte de las unidades agropecuarias se realiza la desparasitación por vía oral 1 vez al año. Las parasitosis de tipo interno son las que más afectan a los bovinos y ovinos sobre todo la fasciolasis. Se reportan otras enfermedades tales como el carbunco. La reproducción no es controlada y los servicios son por monta natural. El mayor problema reproductivo es la retención de placenta en bovinos.

Palabras clave: Unidad agropecuaria, sanidad, reproducción, fasciolasis, parasitosis, carbunco.

${ }^{I}$ Laboratorio de Producción Agropecuaria - FMV - UNMSM.

${ }^{2}$ Laboratorio de Farmacologia- FMV-UNMSM.

${ }^{3}$ Laboratorio de Nutrición-FMV-UNMSM. 


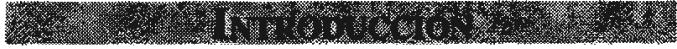

El conocimiento de la realidad es importante para plantear soluciones a los problemas existentes mediante la investigación. Con respecto a los sistemas de producción pecuarios en la sierra de la Provincia de Huaral se conoce muy poco, tanto de los recursos con que cuentan como del potencial que podrían alcanzar; es por ello que poco se puede hacer para proponer programas de diferente índole. Los programas de apoyo pueden ir desde el apoyo técnico directo a los productores hasta la implementación de un Centro Piloto de Investigación, que irradie y difunda los conocimientos técnicos hacia las unidades de producción bajo su área de influencia.

Entre los factores que intervienen en el éxito de una producción pecuaria están la sanidad y la reproducción animal, por lo que el presente estudio tuvo como objetivo conocer la situación actual de la problemática de éstos dos importantes aspectos de la producción pecuaria en la sierra de la provincia de Huaral.

El diagnóstico exploratorio se realizó en 11 de las 35 comunidades campesinas, involucrando de esa manera a seis de los nueve distritos de la Sierra Huaralina.

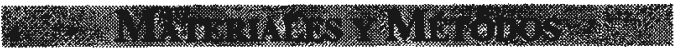

\section{Lugar de estudio}

El lugar de estudio lo conformó la sierra de la provincia de Huaral en el departamento de Lima.

Se consideró como tamaño muestral, el $30 \%$ del total de las comunidades de la zona, lo que dió como resultado la elección de 11 comunidades campesinas.

Las comunidades campesinas seleccionadas para la recolección de datos fue- ron: San Agustín de Huayopampa y Pallac (distrito de Atavillos Bajo) en el Estrato 1. Carac (distrito de 27 de Noviembre), Lampián (distrito de Lampián), San Juan de Uchucuanico y San Cristóbal de Huascoy (distrito de San Miguel de Acos) en el Estrato 2. Viscas, Ravira, Pacaraos y Vichaycocha (distrito de Pacaraos) y Rauma (distrito de Sumbilca) en el Estrato 3.

\section{Método}

Para ajustar la metodología al método de investigación en sistemas agropecuarios, se siguió los siguientes pasos:

\section{- Selección del sistema objetivo y del área de acción}

Los datos se recopilaron de las entidades oficiales como son la Oficina de Información Agraria del Ministerio de Agricultura (OIA-MINAG), la Oficina Agraria Departamental Lima-Callao, Oficina del Servicio Nacional de Sanidad Agraria con sede en Huaral y la Agencia Agraria de Acos. (Borel et al., 1982).

- Recopilación de información y caracterización del manejo sanitario y reproductivo en los sistemas de producción

En esta parte se consideraron tres etapas de trabajo, que son la etapa de visita de reconocimiento, la etapa de instalación y aplicación de las encuestas y la etapa de tabulación y clasificación de la información.

La encuesta se hizo casa por casa, considerando como unidad al comunero que criaba algún tipo de animal con destino al consumo humano. El encargado de responder las preguntas de la encuesta era el comunero, su esposa o el hijo mayor que participaba en el proceso de crianza de los animales. 


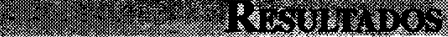

\section{Datos del productor}

La actividad de la producción pecuaria en la sierra de Huaral es dirigida mayoritariamente por los hombres. La mayor parte de los productores agropecuarios de la sierra de Huaral tienen instrucción primaria, y un porcentaje cercano al $30 \%$ posee instrucción secundaria, siendo muy bajo el número de productores con instrucción superior. La mayoría sobrepasa los 50 años de edad.

\section{Descripción del agro-ecosistema}

Un alto porcentaje de productores agropecuarios de los tres estratos de la sierra de Huaral; está asociado a la comunidad, es decir son comuneros.

En cuanto a los porcentajes de distribución de los cultivos en las unidades agropecuarias. En el Estrato 1 (E1) los terrenos destinados exclusivamente a cultivos alimenticios son mayoritarios (82\%), mientras que los destinados a cultivar forrajes son escasos llegando apenas a representar un $2 \%$.

La crianza de los bovinos y ovinos se presenta distribuida en los tres estratos. Es común la crianza de 1 a 10 animales por unidad agropecuaria, que va desde un $46 \%$ (E1) a un $51.9 \%$ (E3) de unidades agropecuarias que crían un número de bovinos dentro de ese rango.

Hay un grupo de productores que posee de 11 a 20 cabezas de ganado bovino, pero este grupo representa desde un $25.9 \%$ (E2) hasta un $31.1 \%$ (E3), siendo escaso el número de productores que poseen de 41 a 50 animales $2 \%$ en el E1 y $0.7 \%$ en el E2. Aunque porcentualmente la crianza de ovinos es mayor en el E1, el número de animales por unidad agropecuaria es de 1 a 10 en un $26 \%$ y de 11 a 20 en un $22 \%$. La crianza de aves, es a escala muy pequeña y con número de animales que van de 1 a 10 en la mayor parte de las unidades agropecuarias que las crían, siendo éstas poco menos al 50\% en cada estrato. Hay un bajo porcentaje de comuneros que crían cerdos predominando más en el E2 pero sin llegar al 50\%. Esta tendencia es similar para los tres estratos.

La asistencia técnica pecuaria a los productores representa un porcentaje que va de un $28.9 \%$ (E2) a un máximo de $46.5 \%$ (E3) de las unidades. Los productores agropecuarios calificaron el desempeño del responsable de brindarles asistencia técnica. Los del E1 consideraron mayoritariamente que la participación del técnico era regular, en tanto que las del E2 determinaron que era regular a buena. Así mismo, la frecuencia de asistencia técnica se realiza en forma esporádica, siendo muy bajo el número de productores que considera que la asistencia es permanente.

Un alto porcentaje de productores agropecuarios realiza controles antiparasitarios de algún tipo. El E2 es el que realiza el menor nivel de control (57.8\%). La gran parte de los productores agropecuarios realiza la desparasitación por vía oral, pues el porcentaje más bajo del uso de este tipo de antiparasitario se observa en el $\mathrm{E} 1$ con un $71.4 \%$. El control antiparasitario mediante baños a los animales es utilizado por una cantidad de productores agropecuarios que bordea el 50\%. Dentro de las parasitosis más frecuentes que afectan a los bovinos de la sierra de Huaral, se observan las de tipo interno son las más frecuentes. En el E1 cobra importancia la fasciolasis y un segundo lugar la asociación de parasitosis intestinal con la fasciolasis. En el E3 la presentación de parasitosis sigue el mismo patrón siendo las de tipo mixtas las que porcentualmente tienen mayor presentación.

El uso de suplemento de minerales y vitaminas extra dietéticas no es una costumbre ni práctica de manejo frecuente en ninguno de los tres estratos. 


\section{Descripción del manejo reproductivo}

En lo que se refiere al manejo reproductivo, menos de la mitad de productores pecuarios detectan o reconocen el celo de sus animales, más del $90 \%$ en los tres estratos no selecciona un macho para que sea el reproductor y sus servicios lo hacen sin control pues los animales se encuentran sueltos, tanto los machos como las hembras, y aún más se encuentran junto a animales de otros productores.

En los tres estratos, los productores reconocen como el mayor problema reproductivo a la retención de placenta $(82.1$ a $87 \%$ ) seguido muy lejos por el aborto, los partos distócicos, y la natimortalidad.

\section{Descripción de las soluciones y expectati- vas del productor}

Los productores agropecuarios demuestran un gran interés por ser parte de una asociación ganadera que los represente, y quizás mediante esa vía encuentren soluciones de sus problemas.

Las expectativas de los productores agropecuarios por recibir algún tipo de apoyo y ayuda técnica está más dirigida al área tanto ganadera como agrícola. Poco son los que manifiestan la necesidad exclusiva del apoyo en el componente ganadero, o unidad agrícola.

Un problema limitante de la producción pecuaria que fue ampliamente reconocido por los productores es la falta de agua, seguido de la ausencia de asesoría técnica especializada.

El diagnóstico estático permite: a) identificar y describir los sistemas de unidades agropecuarias predominantes en el área, b) identificar los sistemas de producción e iniciar su descripción, y c) establecer una es- cala preliminar, jerarquizada, de las limitantes de esos sistemas (CATIE, 1986).

El Grupo social III es el comunero proletario, y es el grupo social predominante en la sierra de la Provincia de Huaral.

Las 35 comunidades campesinas se dividieron, en tres estratos basados en características altitudinales y agro-ecológicas, y se pudo observar características de manejo animal muy similares entre uno y otro estrato. Se concuerda con lo señalado por Gonzales (1973), pues la agricultura y la ganadería son las dos actividades básicas del productor agropecuario de la sierra de Huaral, actividades sobre la cual descansa principalmente su economía y que le ocupan casi la totalidad de su tiempo.

La interacción agricultura-ganadería es de gran magnitud y los cultivos se realizan en sistemas complejos (Quijandría, et al., 1986).

El que los productores llamen técnico a la persona que les da atención a sus animales no dice nada sobre el origen y calidad del conocimiento y experiencia. A esto se suma la inasistencia del apoyo técnico, que hace más difícil el avance de la producción pecuaria en esta parte del país.

La crianza de bovinos y ovinos caracteriza la explotación pecuaria, ya que la crianza de otras especies es para autoabastecimiento, entre ellas en orden de importancia están los cerdos, las aves de corral y los cuyes.

1. Borel, R.; Ruiz, M.; Pezo, D.; Ruiz, A. 1982. Un enfoque metodológico para el desarrollo y evaluación de alternativas de producción pecuaria para el pequeño productor. En Informe del II Taller de Trabajo sobre Sistemas de Producción 
Animal Tropical. Ed. por Li Pun, $\mathrm{H}$. Zandstra, H. Centro Internacional de Investigaciones para el Desarrollo (CIID), Bogotá, Colombia. pp. 41-82.

2. CATIE. 1986. Metodología de la investigación para la generación y desarrollo de alternativas mejoradas en el sistema de producción bovina de doble propósito en Bugaba, Panamá. Centro Agronómico Tropical de Investigación y
Enseñanza. Turrialba, Costa Rica. Informe Técnico $\mathrm{N}^{\circ} 94$, p. 56.

3. Gonzales, S. 1973. Ecología vertical de una cultura de autoabastecimiento a través de Wayllata. Cuzco, Perú.

4. Quijandría, B.; Li Pun, H.; Borel, R., eds. 1986. Informe de la IV Reunión de Trabajo sobre Sistemas de Producción Animal. CIID. Bogotá, Colombia. p. 212. 